\title{
ANALYSIS ON PATH SPACES OVER RIEMANNIAN MANIFOLDS WITH BOUNDARY*
}

\author{
FENG-YU WANG ${ }^{\dagger}$
}

\begin{abstract}
By using Hsu's multiplicative functional for the Neumann heat equation, a natural damped gradient operator is defined for the reflecting Brownian motion on compact manifolds with boundary. This operator is linked to quasi-invariant flows in terms of an integration by parts formula, which leads to the standard log-Sobolev inequality for the associated Dirichlet form on the path space.
\end{abstract}

Key words. Log-Sobolev inequality, integration by parts formula, path space over manifolds with boundary, reflecting Brownian motion.

AMS subject classifications. 60J60, 58G32.

\section{Introduction}

Stochastic analysis on the path space over a complete Riemannian manifold without boundary has been well developed since 1992 when B. K. Driver [3] proved the quasi-invariance theorem for the Brownian motion on compact Riemannian manifolds. A key point of the study is to first establish an integration by parts formula for the associated gradient operator induced by the quasi-invariant flows, and then prove functional inequalities for the corresponding Dirichlet form (see e.g. [5, 9, 2] and references within). For more analysis on Riemannian path spaces we refer to $[4,10,11]$ and references within.

On the other hand, however, the analysis on the path space over a manifold with boundary is still very open. To see this, let us mention [12], where an integration by parts formula was established on the path space of the one-dimensional reflecting Brownian motion. Let e.g. $X_{t}=\left|b_{t}\right|$, where $b_{t}$ is the one-dimensional Brownian motion. For $h \in C([0, T] ; \mathbb{R})$ with $h_{0}=0$ and $\int_{0}^{T}\left|\dot{h}_{t}\right|^{2} \mathrm{~d} t<\infty$, let $\partial_{h}$ be the derivative operator induced by the flow $X+\varepsilon h$, i.e.

$$
\partial_{h} F=\sum_{i=1}^{n} h_{t_{i}} \nabla_{i} f\left(X_{t_{1}}, \cdots, X_{t_{n}}\right),
$$

where $n \in \mathbb{N}, 0<t_{1}<\cdots<t_{n} \leq T$ and $F(X)=f\left(X_{t_{1}}, \cdots, X_{t_{n}}\right)$ for some $f \in C^{\infty}\left(M^{n}\right)$. As the main result of [12], when $h \in C_{0}^{2}(0, T),[12$, Theorem 2.3] provides an integration by parts formula for $\partial_{h}$ by using an infinite-dimensional generalized functional in the sense of Schwartz. Since for non-trivial $h$ the flow is not quasi-invariant, this integration by parts formula can not be formulated by using the distribution of $X$ with a density function, and the induced gradient operator does not provide a Dirichlet form on the $L^{2}$-space of the distribution of $X$. In this paper, we shall establish an essentially different integration by parts formula using quasi-invariant flows.

When $M$ is a half-space of $\mathbb{R}^{d}$, which essentially reduces to the one-dimensional setting, quasi-invariant flows have been constructed in $[1, \S 4(\mathrm{a})]$ by solving SDEs with reflecting boundary. By modifying the idea of [1], we shall define quasi-invariant flows

\footnotetext{
${ }^{*}$ Received: September 8, 2010; accepted (in revised version): June 19, 2011. Communicated by Eric Carlen.

Supported in part by SRFDP and the Fundamental Research Funds for the Central Universities.

†School of Mathematical Sciences, Beijing Normal University, Beijing 100875, China (wangfy@ bnu.edu.cn); Department of Mathematics, Swansea University, Singleton Park, SA2 8PP, UK (F.Y.Wang@swansea.ac.uk).
} 
on a $d$-dimensional Riemannian manifold with boundary for all $h \in \mathbb{H}$ in an intrinsic way, where

$$
\mathbb{H}:=\left\{h \in C\left([0, T] ; \mathbb{R}^{d}\right): h_{0}=0, \int_{0}^{T}\left|\dot{h}_{t}\right|^{2} \mathrm{~d} t<\infty\right\}
$$

is the Cameron-Martin space. By establishing an integration by parts formula, these flows will be linked to a damped gradient operator defined by using Hsu's multiplicative functionals constructed in [9] (see Section 2). In Section 3 we will derive the Gross log-Sobolev inequality for the associated Dirichlet form.

\section{Damped gradient and integration by parts}

In this section, we aim to define the damped gradient operator by using the multiplicative functional constructed in [9] to replace the known one as in [7] on manifolds without boundary, and to link this operator to quasi-invariant flows constructed by solving SDEs with reflection as in [1] where the half-space of $\mathbb{R}^{d}$ is considered. To this end, we first recall the construction of the reflecting Brownian motion by solving SDEs on the manifold with local times, then introduce Hsu's multiplicative functional which gives an explicit formulation of the damped gradient for cylindrical functions, and finally establish an integration by parts formula which links the gradient operator to quasi-invariant flows constructed in the spirit of [1].

Let $M$ be a $d$-dimensional compact connected Riemannian manifold with boundary $\partial M$. Let $o \in M$ and $T>0$ be fixed. Then the path space for the reflecting Brownian motion on $M$ starting at $o$ is

$$
W=\left\{\gamma \in C([0, T] ; M): \gamma_{0}=o\right\} .
$$

Let $B_{t}$ be the $d$-dimensional Brownian motion on a complete probability space $(\Omega, \mathcal{F}, \mathbb{P})$ with natural filtration $\left\{\mathcal{F}_{t}\right\}_{t \geq 0}$. For any $x \in M$, let $O_{x} M$ be the set of all orthonormal bases for the tangent space $T_{x} M$ at point $x$, and let $O(M):=\cup_{x \in M} O_{x}(M)$ be the frame bundle. Then for any $x \in M$, the reflecting Brownian motion starting at $x$ can be constructed by solving the SDE

$$
\mathrm{d} X_{t}^{x}=u_{t}^{x} \circ \mathrm{d} B_{t}+N\left(X_{t}^{x}\right) \mathrm{d} l_{t}^{x},
$$

where $u_{t}^{x} \in O_{X_{t}^{x}}(M)$ is the horizontal lift of $X_{t}^{x}$ on the frame bundle $O(M), N$ is the inward unit normal vector field on $\partial M$, and $l_{t}^{x}$ is the local time of $X_{t}^{x}$ on the boundary $\partial M$. Let $X^{x}=\left\{X_{t}^{x}: 0 \leq t \leq T\right\}$.

To introduce Hsu's multiplicative functional, we need to introduce some $\mathbb{R}^{d} \otimes \mathbb{R}^{d_{-}}$ valued functionals on the frame bundle. Let Ric be the Ricci curvature on $M$ and $\mathbb{I}$ the second fundamental form on $\partial M$. For any $u \in O(M)$, let

$$
R_{u}(a, b)=\operatorname{Ric}(u a, u b), \quad a, b \in \mathbb{R}^{d} .
$$

Let $\pi_{\partial}: T M \rightarrow T \partial M$ be the orthogonal projection at points on $\partial M$, and let $\pi: O(M) \rightarrow$ $M$ is the canonical projection. For any $u \in O(M)$ with $\pi u \in \partial M$, let

$$
\mathbb{I}_{u}(a, b)=\mathbb{I}\left(\pi_{\partial} u a, \pi_{\partial} u b\right), \quad a, b \in \mathbb{R}^{d} .
$$

Finally, for $u \in \partial O(M)$, let

$$
P_{u}(a, b)=\langle u a, N\rangle\langle u b, N\rangle, \quad a, b \in \mathbb{R}^{d} .
$$


For any $\varepsilon>0$, let $Q_{t}^{\varepsilon}$ solve the following $\mathrm{SDE}$ on $\mathbb{R}^{d} \otimes \mathbb{R}^{d}$ :

$$
\mathrm{d} Q_{t}^{x, \varepsilon}=-Q_{t}^{x, \varepsilon}\left\{\frac{1}{2} R_{u_{t}^{x}} \mathrm{~d} t+\left(\varepsilon^{-1} P_{u_{t}^{x}}+\mathbb{I}_{u_{t}^{x}}\right) \mathrm{d} l_{t}\right\}, \quad Q_{0}^{x, \varepsilon}=I .
$$

According to [9, Theorem 3.4], when $\varepsilon \downarrow 0$ the process $Q_{t}^{x, \varepsilon}$ converges in $L^{2}$ to an adapted right-continuous process $Q_{t}^{x}$ with left limit, such that $Q_{t}^{x} P_{u_{t}^{x}}=0$ if $X_{t}^{x} \in \partial M$. Consequently, if Ric $\geq-K$ and $\mathbb{I} \geq-\sigma$ for some continuous functions $K$ and $\sigma$ on $M$, then

$$
\left\|Q_{t}^{x}\right\| \leq \exp \left\{\frac{1}{2} \int_{0}^{t} K\left(X_{s}^{x}\right) \mathrm{d} s+\int_{0}^{t} \sigma\left(X_{s}^{x}\right) \mathrm{d} l_{s}^{x}\right\}, t \geq 0,
$$

where $\|\cdot\|$ is the operator norm on $\mathbb{R}^{d}$. In particular, $\mathbb{E}\left\|Q_{t}^{x}\right\|^{p}<\infty$ holds for any $p>1$. For $f \in C(M)$, let

$$
P_{t} f(x)=\mathbb{E} f\left(X_{t}^{x}\right), \quad x \in M .
$$

Then $P_{t}$ is the Neumann semigroup. By [9, Theorem 4.2] (see also the last display in the proof of [9, Theorem 5.1]), $s \mapsto Q_{s}^{x}\left(u_{s}^{x}\right)^{-1} \nabla P_{t-s} f\left(X_{s}^{x}\right)$ is a martingale. So,

$$
\left(u_{0}^{x}\right)^{-1} \nabla P_{t} f(x)=\mathbb{E}\left\{Q_{t}^{x}\left(u_{t}^{x}\right)^{-1} \nabla f\left(X_{t}^{x}\right)\right\}, \quad x \in M, u_{0} \in O_{x}(M) .
$$

In general, for $s \geq 0$, let $\left(Q_{s, t+s}^{x}\right)_{t \geq 0}$ be the associated multiplicative functional for the process $\left(X_{t+s}^{x}\right)_{t \geq 0}$. We have

$$
Q_{s, t}^{x} Q_{t, r}^{x}=Q_{s, r}^{x}, \quad 0 \leq s \leq t \leq r .
$$

We shall use these multiplicative functionals to define the damped gradient operator (see [7] for the damped gradient operator for manifolds without boundary).

Let

$$
\mathcal{F} C^{\infty}=\left\{W \ni \gamma \mapsto f\left(\gamma_{t_{1}}, \cdots, \gamma_{t_{n}}\right): n \geq 1,0<t_{1}<\cdots<t_{n} \leq T, f \in C^{\infty}(M)\right\}
$$

be the class of smooth cylindrical functions on $W$. For any $F \in \mathcal{F} C^{\infty}$ with $F(\gamma)=$ $f\left(\gamma_{t_{1}}, \cdots, \gamma_{t_{n}}\right)$, define the damped gradient $D F\left(X^{x}\right)$ as an $\mathbb{H}$-valued random variable by setting $(D F)_{0}\left(X^{x}\right)=0$ and

$$
\frac{\mathrm{d}}{\mathrm{d} t}(D F)_{t}\left(X^{x}\right)=\sum_{i=1}^{n} 1_{\left\{t<t_{i}\right\}} Q_{t, t_{i}}^{x}\left(u_{t_{i}}^{x}\right)^{-1} \nabla_{i} f\left(X_{t_{1}}^{x}, \cdots, X_{t_{n}}^{x}\right), \quad t \in[0, T],
$$

where $\nabla_{i}$ denotes the gradient operator with respect to the $i$-th component. Then, for any $\mathbb{H}$-valued random variable $h$, we have

$$
D_{h} F\left(X^{x}\right):=\left\langle D F\left(X^{x}\right), h\right\rangle_{\mathbb{H}}=\sum_{i=1}^{n} \int_{0}^{t_{i}}\left\langle\left(u_{t_{i}}^{x}\right)^{-1} \nabla_{i} f\left(X_{t_{1}}^{x}, \cdots, X_{t_{n}}^{x}\right),\left(Q_{t, t_{i}}^{x}\right)^{*} \dot{h}_{t}\right\rangle \mathrm{d} t .
$$

Note that when $\partial M=\emptyset$, we may let $l_{t} \equiv 0$ in $(2.2)$, so that our formulation of $D_{h} F$ goes back to the known one presented in [7] for manifolds without boundary.

Now, we intend to link $D_{h} F$ to the directional derivative induced by a quasiinvariant flow. Let $\tilde{\mathbb{H}}$ denote the set of all square-integrable $\mathbb{H}$-valued adapted random variables, i.e.

$$
\tilde{\mathbb{H}}=\left\{h \in L^{2}(\Omega \rightarrow \mathbb{H} ; \mathbb{P}): h_{t} \text { is } \mathcal{F}_{t^{-}} \text {-measurable, } t \in[0, T]\right\} .
$$


Then $\tilde{\mathbb{H}}$ is a Hilbert space with inner product

$$
\langle h, \tilde{h}\rangle_{\tilde{\mathbb{H}}}:=\mathbb{E} \int_{0}^{T}\left\langle\dot{h}_{t}, \dot{\tilde{h}}_{t}\right\rangle \mathrm{d} t=\mathbb{E}\langle h, \tilde{h}\rangle_{\mathbb{H}}, \quad h, \tilde{h} \in \tilde{\mathbb{H}} .
$$

To describe $D F$ by using a quasi-invariant flow, for $h \in \tilde{\mathbb{H}}$ and $\varepsilon>0$ let $X_{t}^{\varepsilon, h}$ solve the SDE

$$
\mathrm{d} X_{t}^{\varepsilon, h}=u_{t}^{\varepsilon, h} \circ \mathrm{d} B_{t}+N\left(X_{t}^{\varepsilon, h}\right) \mathrm{d} l_{t}^{\varepsilon, h}+\varepsilon u_{t}^{\varepsilon, h} \dot{h}_{t} \mathrm{~d} t, \quad X_{0}^{\varepsilon, h}=x=\pi u_{0}^{x},
$$

where $l_{t}^{\varepsilon, h}$ and $u_{t}^{\varepsilon, h}$ are, respectively, the local time on $\partial M$ and the horizontal lift on $O(M)$ for $X_{t}^{\varepsilon, h}$. Recall that when $M$ is a half-space of $\mathbb{R}^{d}$, it is shown in [1] that $\left\{X^{\varepsilon, h}\right\}_{\varepsilon \geq 0}$ is a quasi-invariant flow. To see that $\left\{X^{\varepsilon, h}\right\}_{\varepsilon \geq 0}$ has the flow property also in the Riemannian setting, let

$$
\Theta: W_{0}:=\left\{\omega \in C([0, T]): \omega_{0}=0\right\} \rightarrow W
$$

be measurable such that $X=\Theta(B)$. For any $\varepsilon>0$ and a function $\Phi: W_{0} \rightarrow W$, let $\left(\theta_{\varepsilon}^{h} \Phi\right)(\omega)=\Phi(\omega+\varepsilon h)$. Then $X^{\varepsilon, h}=\left(\theta_{\varepsilon}^{h} \Theta\right)(B), \varepsilon \geq 0$. Hence,

$$
X^{\varepsilon_{1}+\varepsilon_{2}, h}=\theta_{\varepsilon_{1}}^{h} X^{\varepsilon_{2}, h}, \quad \varepsilon_{1}, \varepsilon_{2} \geq 0 .
$$

Moreover, let us explain that the flow is quasi-invariant, i.e. for each $\varepsilon \geq 0$, the distribution of $X^{\varepsilon, h}$ is absolutely continuous with respect to that of $X$. Let

$$
R^{\varepsilon, h}=\exp \left[\varepsilon \int_{0}^{T}\left\langle\dot{h}_{t}, \mathrm{~d} B_{t}\right\rangle-\frac{\varepsilon^{2}}{2} \int_{0}^{T}\left|\dot{h}_{t}\right|^{2} \mathrm{~d} t\right] .
$$

By the Girsanov theorem,

$$
B_{t}^{\varepsilon, h}:=B_{t}-\varepsilon h_{t}
$$

is the $d$-dimensional Brownian motion under the probability $R^{\varepsilon, h} \mathbb{P}$. Thus, the distribution of $X$ under $R^{\varepsilon, h} \mathbb{P}$ coincides with that of $X^{\varepsilon, h}$ under $\mathbb{P}$. Therefore, $X^{\varepsilon, h}$ is quasi-invariant.

Finally, we are able to introduce the main result in this section, which provides an integration by parts formula for $D_{h}$ as well as a link between the damped gradient $D_{h}$ and the directional derivative induced by the flow $\left\{X^{\varepsilon, h}\right\}_{\varepsilon \geq 0}$.

Theorem 2.1. For any $x \in M$ and $F \in \mathcal{F} C^{\infty}$,

$$
\mathbb{E}\left\{D_{h} F\right\}\left(X^{x}\right)=\lim _{\varepsilon \downarrow 0} \mathbb{E} \frac{F\left(X^{\varepsilon, h}\right)-F\left(X^{x}\right)}{\varepsilon}=\mathbb{E}\left\{F\left(X^{x}\right) \int_{0}^{T}\left\langle\dot{h}_{t}, \mathrm{~d} B_{t}\right\rangle\right\}
$$

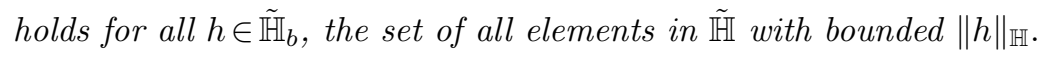

Since $\tilde{\mathbb{H}}_{b}$ is dense in $\tilde{\mathbb{H}}$, the above result implies that the projection of $D$ onto $\tilde{\mathbb{H}}$ can be determined by the flows $X^{\varepsilon, h}, h \in \tilde{\mathbb{H}}_{b}$. But it is not clear whether

$$
D_{h} F\left(X^{x}\right)=\lim _{\varepsilon \downarrow 0} \frac{F\left(X^{\varepsilon, h}\right)-F\left(X^{x}\right)}{\varepsilon}, h \in \tilde{\mathbb{H}}
$$

holds or not. 
To prove Theorem 2.1, we need some preparations. In particular, we shall use (2.3) and a conducting argument as in [8] for the case without boundary.

Lemma 2.2. Let $x \in M$ and $F \in \mathcal{F} C^{\infty}$. Then

$$
\lim _{\varepsilon \downarrow 0} \mathbb{E} \frac{F\left(X^{\varepsilon, h}\right)-F\left(X^{x}\right)}{\varepsilon}=\mathbb{E}\left\{F\left(X^{x}\right) \int_{0}^{T}\left\langle\dot{h}_{t}, \mathrm{~d} B_{t}\right\rangle\right\}
$$

holds for all $h \in \tilde{\mathbb{H}}_{b}$.

Proof. Let $B_{t}^{\varepsilon, h}=B_{t}-\varepsilon h_{t}$, which is the $d$-dimensional Brownian motion under $R^{\varepsilon, h} \mathbb{P}$. Reformulate (2.1) as

$$
\mathrm{d} X_{t}^{x}=u_{t}^{x} \circ \mathrm{d} B_{t}^{\varepsilon, h}+N\left(X_{t}^{x}\right) \mathrm{d} l_{t}^{x}+\varepsilon u_{t} \dot{h}_{t} \mathrm{~d} t .
$$

By the weak uniqueness of (2.6), we conclude that the distribution of $X^{x}$ under $R^{\varepsilon, h} \mathbb{P}$ coincides with that of $X^{\varepsilon, h}$ under $\mathbb{P}$. In particular, $\mathbb{E} F\left(X^{\varepsilon, h}\right)=\mathbb{E}\left[R^{\varepsilon, h} F\left(X^{x}\right)\right]$. Thus,

$$
\begin{aligned}
& \lim _{\varepsilon \downarrow 0} \mathbb{E} \frac{F\left(X^{\varepsilon, h}\right)-F\left(X^{x}\right)}{\varepsilon}=\lim _{\varepsilon \downarrow 0} \mathbb{E}\left\{F\left(X^{x}\right) \cdot \frac{R^{\varepsilon, h}-1}{\varepsilon}\right\} \\
= & \mathbb{E}\left\{F\left(X^{x}\right) \int_{0}^{T}\left\langle\dot{h}_{t}, \mathrm{~d} B_{t}\right\rangle\right\},
\end{aligned}
$$

where the last step is due to the dominated convergence theorem since $\left\{R^{\varepsilon, h}\right\}_{\varepsilon \in[0,1]}$ is uniformly integrable for $h \in \tilde{\mathbb{H}}_{b}$.

Lemma 2.3. For any $n \geq 1,0<t_{1}<\cdots<t_{n} \leq T$, and $f \in C^{\infty}\left(M^{n}\right)$,

$$
\left(u_{0}^{x}\right)^{-1} \nabla_{x} \mathbb{E} f\left(X_{t_{1}}^{x}, \cdots, X_{t_{n}}^{x}\right)=\sum_{i=1}^{n} \mathbb{E}\left\{Q_{t_{i}}^{x}\left(u_{t_{i}}^{x}\right)^{-1} \nabla_{i} f\left(X_{t_{1}}^{x}, \cdots, X_{t_{n}}^{x}\right)\right\}
$$

holds for all $x \in M$ and $u_{0}^{x} \in O_{x}(M)$, where $\nabla_{x}$ denotes the gradient with respect to $x$.

Proof. By (2.3), the desired assertion holds for $n=1$. Assume that it holds for $n=k$ for some natural number $k \geq 1$. It remains to prove the assertion for $n=k+1$. To this end, set

$$
g(x)=\mathbb{E} f\left(x, X_{t_{2}-t_{1}}^{x}, \cdots, X_{t_{k+1}-t_{1}}^{x}\right), \quad x \in M .
$$

By the assumption for $n=k$ we have

$$
\left(u_{0}^{x}\right)^{-1} \nabla g(x)=\sum_{i=1}^{k+1} \mathbb{E}\left\{Q_{t_{i}-t_{1}}^{x}\left(u_{t_{i}-t_{1}}^{x}\right)^{-1} \nabla_{i} f\left(x, X_{t_{2}-t_{1}}^{x}, \cdots, X_{t_{k+1}-t_{1}}^{x}\right)\right\}
$$

for all $x \in M, u_{0} \in O_{x}(M)$. Combining this with the assertion for $k=1$ and using the Markov property, we obtain

$$
\begin{aligned}
& \left(u_{0}^{x}\right)^{-1} \nabla_{x} \mathbb{E} f\left(X_{t_{1}}^{x}, \cdots, X_{t_{k+1}}^{x}\right)=\left(u_{0}^{x}\right)^{-1} \nabla_{x} \mathbb{E} g\left(X_{t_{1}}^{x}\right) \\
= & \mathbb{E}\left\{Q_{t_{1}}^{x}\left(u_{t_{1}}^{x}\right)^{-1} \nabla g\left(X_{t_{1}}^{x}\right)\right\}=\sum_{i=1}^{k+1} \mathbb{E}\left\{Q_{t_{i}}^{x}\left(u_{t_{i}}^{x}\right)^{-1} \nabla_{i} f\left(X_{t_{1}}^{x}, \cdots, X_{t_{k+1}}^{x}\right)\right\} .
\end{aligned}
$$


Lemma 2.4. Let $f \in C^{\infty}(M)$. Then for any $x \in M$ and $t>0$,

$$
\mathbb{E}\left\{f\left(X_{t}^{x}\right) \int_{0}^{t}\left\langle\dot{h}_{s}, \mathrm{~d} B_{s}\right\rangle\right\}=\mathbb{E} \int_{0}^{t}\left\langle\left(u_{t}^{x}\right)^{-1} \nabla f\left(X_{t}^{x}\right),\left(Q_{s, t}^{x}\right)^{*} \dot{h}_{s}\right\rangle \mathrm{d} s, \quad h \in \tilde{\mathbb{H}}, t_{1} \in[0, T] .
$$

Proof. Noting that

$$
\frac{\mathrm{d}}{\mathrm{d} s} P_{s} f=\frac{1}{2} \Delta P_{s} f,\left.\quad N P_{s} f\right|_{\partial M}=0, \quad s>0,
$$

by (2.1) and the Itô formula we obtain

$$
\mathrm{d}\left(P_{t-s} f\right)\left(X_{s}^{x}\right)=\left\langle\nabla P_{t-s} f\left(X_{s}^{x}\right), u_{s}^{x} \mathrm{~d} B_{s}\right\rangle, \quad s \in[0, t) .
$$

This implies

$$
f\left(X_{t}^{x}\right)=P_{t} f(x)+\int_{0}^{t}\left\langle\left(u_{s}^{x}\right)^{-1} \nabla P_{t-s} f\left(X_{s}^{x}\right), \mathrm{d} B_{s}\right\rangle, \quad s \in[0, t] .
$$

Therefore,

$$
\mathbb{E}\left\{f\left(X_{t}^{x}\right) \int_{0}^{t}\left\langle\dot{h}_{s}, \mathrm{~d} B_{s}\right\rangle\right\}=\mathbb{E} \int_{0}^{t}\left\langle\left(u_{s}^{x}\right)^{-1} \nabla P_{t-s} f\left(X_{s}^{x}\right), \dot{h}_{s}\right\rangle \mathrm{d} s .
$$

By (2.3) and the Markov property we have

$$
\left(u_{s}^{x}\right)^{-1} \nabla P_{t-s} f\left(X_{s}^{x}\right)=\mathbb{E}\left(Q_{s, t}^{x}\left(u_{t}^{x}\right)^{-1} \nabla f\left(X_{t}^{x}\right) \mid \mathcal{F}_{s}\right) .
$$

So, the desired formula follows from (2.8) since $\dot{h}_{s}$ is $\mathcal{F}_{s}$-measurable.

As a consequence of (2.3) and Lemma 2.4, we have the following Bismut formula.

Corollary 2.5. For any $v \in T_{x} M$ and any $h \in \tilde{H}$ with $h_{t}=\left(u_{0}^{x}\right)^{-1} v$,

$$
\left\langle v, \nabla P_{t} f(x)\right\rangle=\mathbb{E}\left\{f\left(X_{t}^{x}\right) \int_{0}^{t}\left\langle\left(Q_{s}^{x}\right)^{*} \dot{h}_{s}, \mathrm{~d} B_{s}\right\rangle\right\} .
$$

Proof. By (2.4) and applying Lemma 2.4 to $\tilde{h} \in \tilde{\mathbb{H}}$ in place of $h$, where $\dot{\tilde{h}}_{s}=$ $\left(Q_{s}^{x}\right)^{*} \dot{h}_{s}$, we obtain

$$
\begin{aligned}
\mathbb{E}\left\{f\left(X_{t}\right) \int_{0}^{t}\left\langle\left(Q_{s}^{x}\right)^{*} \dot{h}_{s}, \mathrm{~d} B_{s}\right\rangle\right\} & =\mathbb{E} \int_{0}^{t}\left\langle\left(u_{t}^{x}\right)^{-1} \nabla f\left(X_{t}^{x}\right),\left(Q_{t}^{x}\right)^{*} \dot{h}_{s}\right\rangle \mathrm{d} s \\
& =\mathbb{E}\left\langle Q_{t}^{x}\left(u_{t}^{x}\right)^{-1} \nabla f\left(X_{t}\right),\left(u_{0}^{x}\right)^{-1} v\right\rangle
\end{aligned}
$$

Then the proof is completed by combining this with (2.3).

Proof. [Proof of Theorem 2.1.] By Lemma 2.2, it suffices to prove

$$
\mathbb{E}\left\{D_{h} F\right\}\left(X^{x}\right)=\mathbb{E}\left\{F\left(X^{x}\right) \int_{0}^{T}\left\langle\dot{h}_{t}, \mathrm{~d} B_{t}\right\rangle\right\}, \quad h \in \tilde{\mathbb{H}}
$$


for $F\left(X^{x}\right)=f\left(X_{t_{1}}^{x}, \cdots, X_{t_{n}}^{x}\right)$ with $f \in C^{\infty}\left(M^{n}\right)$, where $n \geq 1,0<t_{1}<\cdots<t_{n} \leq T$. According to Lemma 2.4, (2.9) holds for $n=1$. Assuming (2.9) holds for $n=k$ for some $k \geq 1$, we aim to prove it for $n=k+1$. To this end, let

$$
g(x)=\mathbb{E} f\left(x, X_{t_{2}-t_{1}}^{x}, \cdots, X_{t_{k+1}-t_{1}}^{x}\right), \quad x \in M .
$$

By the result for $n=1$ and the Markov property,

$$
\begin{aligned}
& \int_{0}^{t_{1}} \mathbb{E}\left\langle\left(u_{t_{1}}^{x}\right)^{-1} \nabla g\left(X_{t_{1}}^{x}\right),\left(Q_{t, t_{1}}^{x}\right)^{*} \dot{h}_{t}\right\rangle \mathrm{d} t \\
= & \mathbb{E}\left\{\mathbb{E}\left(F\left(X^{x}\right) \mid \mathcal{F}_{t_{1}}\right) \int_{0}^{t_{1}}\left\langle\dot{h}_{t}, \mathrm{~d} B_{t}\right\rangle\right\}=\mathbb{E}\left\{F\left(X^{x}\right) \int_{0}^{t_{1}}\left\langle\dot{h}_{t}, \mathrm{~d} B_{t}\right\rangle\right\} .
\end{aligned}
$$

On the other hand, by (2.4), Lemma 2.3, and the Markov property,

$$
\begin{aligned}
& \int_{0}^{t_{1}} \mathbb{E}\left\langle\left(u_{t_{1}}^{x}\right)^{-1} \nabla g\left(X_{t_{1}}^{x}\right),\left(Q_{t, t_{1}}^{x}\right)^{*} \dot{h}_{t}\right\rangle \mathrm{d} t \\
= & \int_{0}^{t_{1}} \mathbb{E}\left\langle\mathbb{E}\left(\sum_{i=1}^{k+1} Q_{t_{1}, t_{i}}^{x}\left(u_{t_{i}}^{x}\right)^{-1} \nabla_{i} f\left(X_{t_{1}}^{x}, \cdots, X_{t_{k+1}}^{x}\right) \mid \mathcal{F}_{t_{1}}\right),\left(Q_{t, t_{1}}^{x}\right)^{*} \dot{h}_{t}\right\rangle \mathrm{d} t \\
= & \sum_{i=1}^{k+1} \int_{0}^{t_{1}}\left\langle\left(u_{t_{i}}^{x}\right)^{-1} \nabla_{i} f\left(X_{t_{1}}^{x}, \cdots, X_{t_{k+1}}^{x}\right),\left(Q_{t, t_{i}}^{x}\right)^{*} \dot{h}_{t}\right\rangle \mathrm{d} t .
\end{aligned}
$$

Combining this with (2.5) and (2.10) we obtain

$$
\begin{aligned}
\mathbb{E}\left\{D_{h} F\left(X^{x}\right)\right\}= & \mathbb{E}\left\{F\left(X^{x}\right) \int_{0}^{t_{1}}\left\langle\dot{h}_{t}, \mathrm{~d} B_{t}\right\rangle\right\} \\
& +\mathbb{E} \sum_{i=2}^{k+1} \int_{t_{1}}^{t_{i}}\left\langle\left(u_{t_{i}}^{x}\right)^{-1} \nabla_{i} f\left(X_{t_{1}}^{x}, \cdots, X_{t_{k+1}}^{x}\right),\left(Q_{t, t_{i}}^{x}\right)^{*} \dot{h}_{t}\right\rangle \mathrm{d} t .
\end{aligned}
$$

By the Markov property and the assumption for $n=k$, we have

$$
\sum_{i=2}^{k+1} \mathbb{E} \int_{t_{1}}^{t_{i}}\left\langle\left(u_{t_{i}}^{x}\right)^{-1} \nabla_{i} f\left(X_{t_{1}}^{x}, \cdots, X_{t_{k+1}}^{x}\right),\left(Q_{t, t_{i}}^{x}\right)^{*} \dot{h}_{t}\right\rangle \mathrm{d} t=\mathbb{E}\left\{F\left(X^{x}\right) \int_{t_{1}}^{T}\left\langle\dot{h}_{t}, \mathrm{~d} B_{t}\right\rangle\right\} .
$$

Combining this with (2.11) we complete the proof.

\section{The log-Sobolev inequality}

Let $\mu$ be the distribution of $X:=X^{o}$ for a fixed point $o \in M$. Let

$$
\mathcal{E}(F, G)=\mathbb{E}\left\{\langle D F, D G\rangle_{\mathbb{H}}(X)\right\}, \quad F, G \in \mathcal{F} C^{\infty}
$$

Since both $D F$ and $D G$ are functionals of $X,\left(\mathcal{E}, \mathcal{F} C^{\infty}\right)$ is a positive bilinear form on $L^{2}(W ; \mu)$. It is standard that the integration by parts formula $(2.9)$ implies the closability of the form (see Lemma 3.1). We shall use $(\mathcal{E}, \mathcal{D}(\mathcal{E})$ ) to denote the closure of $\left(\mathcal{E}, \mathcal{F} C^{\infty}\right)$. Moreover, (2.9) also implies the Clark-Ocone type martingale representation formula (see Lemma 3.2), which leads to the standard Gross [6] log-Sobolev inequality. It is well known that the log-Sobolev inequality implies that the associated Markov semigroup is hypercontractive and converges exponentially to $\mu$ in the sense of relative entropy. 
Lemma 3.1. $\left(\mathcal{E}, \mathcal{F} C^{\infty}\right)$ is closable in $L^{2}(W ; \mu)$.

Proof. Although the proof is standard by using the integration by parts formula, we include it here for completeness. Let $\left\{F_{n}\right\}_{n \geq 1} \subset \mathcal{F} C^{\infty}$ such that $\mathcal{E}\left(F_{n}, F_{n}\right) \leq 1$ for all $n \geq 0$ and $\mu\left(F_{n}^{2}\right)+\mathcal{E}\left(F_{n}-F_{m}, F_{n}-F_{m}\right) \rightarrow 0$ as $n, m \rightarrow \infty$. We aim to prove that $\mathcal{E}\left(F_{n}, F_{n}\right) \rightarrow 0$ as $n \rightarrow \infty$. Since

$$
\mathcal{E}\left(F_{n}, F_{n}\right)=\mathcal{E}\left(F_{n}, F_{n}-F_{m}\right)+\mathcal{E}\left(F_{n}, F_{m}\right) \leq \sqrt{\mathcal{E}\left(F_{n}-F_{m}, F_{n}-F_{m}\right)}+\mathcal{E}\left(F_{n}, F_{m}\right),
$$

it suffices to show that for any $G \in \mathcal{F} C^{\infty}$, one has $\mathcal{E}\left(F_{n}, G\right) \rightarrow 0$ as $n \rightarrow \infty$. To this end, let $\left\{h^{i}\right\}_{i \geq 1}$ be an ONB on $\mathbb{H}$. For any $\varepsilon>0$ there exists $k \geq 1$ such that

$$
\left|\mathcal{E}\left(F_{n}, G\right)-\sum_{i=1}^{k} \mathbb{E}\left\{\left(D_{h^{i}} F_{n}\right)\left(D_{h^{i}} G\right)\right\}(X)\right|<\varepsilon,
$$

where $D_{h} F:=\langle D F, h\rangle_{\mathbb{H}}$ for $F \in \mathcal{F} C^{\infty}$ and $h \in \mathbb{H}$. Since $\mathcal{F} C^{\infty}$ is dense in $L^{2}(W ; \mu)$, there exists $G_{i} \in \mathcal{F} C^{\infty}$ such that

$$
\mathbb{E}\left\{\left|D_{h^{i}} G-G_{i}\right|^{2}(X)\right\}<\varepsilon, \quad 1 \leq i \leq k .
$$

Therefore,

$$
\left|\mathcal{E}\left(F_{n}, G\right)\right| \leq 2 \varepsilon+\sum_{i=1}^{k}\left|\mathbb{E}\left\langle\left(G_{i} D F_{n}\right)(X), h_{i}\right\rangle_{\mathbb{H}}\right| .
$$

Noting that $G_{i} D F_{n}=D\left(F_{n} G_{i}\right)-F_{n} D G_{i}$, by $(2.9)$ we obtain

$$
\left|\mathcal{E}\left(F_{n}, G\right)\right| \leq 2 \varepsilon+\sum_{i=1}^{k}\left|\mathbb{E}\left[F_{n}(X)\left\{G_{i}(X) \int_{0}^{T}\left\langle\dot{h}_{t}^{i}, \mathrm{~d} B_{t}\right\rangle-D_{h^{i}} G_{i}(X)\right\}\right]\right| .
$$

Since $\mu\left(F_{n}^{2}\right) \rightarrow 0$ as $n \rightarrow \infty$, by letting first $n \rightarrow \infty$ and then $\varepsilon \rightarrow 0$ we complete the proof.

Lemma 3.2. For any $F \in \mathcal{F} C^{\infty}$, let $\tilde{D} F(X)$ be the projection of $D F(X)$ on $\tilde{\mathbb{H}}$, i.e.

$$
\frac{\mathrm{d}}{\mathrm{d} t}(\tilde{D} F)_{t}(X)=\mathbb{E}\left(\frac{\mathrm{d}}{\mathrm{d} t}(D F)_{t}(X) \mid \mathcal{F}_{t}\right), \quad t \in[0, T],(\tilde{D} F)_{0}=0 .
$$

Then

$$
F(X)=\mathbb{E} F(X)+\int_{0}^{T}\left\langle\frac{\mathrm{d}}{\mathrm{d} t}(\tilde{D} F)_{t}(X), \mathrm{d} B_{t}\right\rangle
$$

Proof. By Theorem 2.1, we have

$$
\mathbb{E}\langle h, \tilde{D} F\rangle_{\mathbb{H}}(X)=\mathbb{E}\left\{F(X) \int_{0}^{T}\left\langle\dot{h}_{t}, \mathrm{~d} B_{t}\right\rangle\right\}, \quad h \in \tilde{\mathbb{H}} .
$$

On the other hand, by the martingale representation, there exists a predictable process $\beta_{t}$ such that

$$
\mathbb{E}\left(F(X) \mid \mathcal{F}_{t}\right)=\mathbb{E} F(X)+\int_{0}^{t}\left\langle\beta_{s}, \mathrm{~d} B_{s}\right\rangle, \quad t \in[0, T]
$$


Let

$$
\varphi_{t}=\int_{0}^{t} \beta_{s} \mathrm{~d} s, \quad t \in[0, T]
$$

We have $\varphi \in \tilde{\mathbb{H}}$ and, by (3.2),

$$
\mathbb{E}\langle h, \varphi\rangle_{\mathbb{H}}=\mathbb{E} \int_{0}^{T}\left\langle\dot{h}_{t}, \beta_{t}\right\rangle \mathrm{d} t=\mathbb{E}\left\{F(X) \int_{0}^{T}\left\langle\dot{h}_{t}, \mathrm{~d} B_{t}\right\rangle\right\}
$$

holds for all $h \in \tilde{\mathbb{H}}$. Combining this with $(3.1)$ we conclude that $\tilde{D} F(X)=\varphi$. Therefore, the desired formula follows from (3.2).

It is standard that the martingale representation in Lemma 3.2 implies the following $\log$-Sobolev inequality. Since the parameter $T$ has been properly contained in the Dirichlet form $\mathbb{E}$ just as in the case without boundary (see [7]), the resulting $\log$-Sobolev constant is independent of $T$. Moreover, since it is well-known that the constant 2 in the inequality is $\operatorname{sharp}$ for $M=\mathbb{R}^{d}$, it is also sharp as a universal constant for compact manifolds with boundary as $\mathbb{R}^{d}$ can be approximated by bounded balls.

Theorem 3.3. For any $T>0$ and $o \in M,(\mathcal{E}, \mathcal{D}(\mathcal{E}))$ satisfies the following log-Sobolev inequality:

$$
\mu\left(F^{2} \log F^{2}\right) \leq 2 \mathcal{E}(F, F), \quad F \in \mathcal{D}(\mathcal{E}), \mu\left(F^{2}\right)=1 .
$$

Proof. It suffices to prove the inequality for $F \in \mathcal{F} C^{\infty}$. Let $m_{t}=\mathbb{E}\left(F(X)^{2} \mid \mathcal{F}_{t}\right), t \in$ $[0, T]$. By Lemma 3.2 and the Itô formula,

$$
\mathrm{d} m_{t} \log m_{t}=\left(1+\log m_{t}\right) \mathrm{d} m_{t}+\frac{\left|\frac{\mathrm{d}}{\mathrm{d} t}\left(\tilde{D} F^{2}\right)_{t}(X)\right|^{2}}{2 m_{t}} \mathrm{~d} t .
$$

Thus,

$$
\begin{aligned}
\mu\left(F^{2} \log F^{2}\right) & =\mathbb{E} m_{T} \log m_{T}=\int_{0}^{T} \frac{2 \mathbb{E}\left(F(X) \frac{\mathrm{d}}{\mathrm{d} t}(D F)_{t}(X) \mid \mathcal{F}_{t}\right)^{2}}{\mathbb{E}\left(F(X)^{2} \mid \mathcal{F}_{t}\right)} \mathrm{d} t \\
& \leq 2 \int_{0}^{T} \mathbb{E}\left|\frac{\mathrm{d}}{\mathrm{d} t}(D F)_{t}(X)\right|^{2} \mathrm{~d} t=2 \mathbb{E}\|D F(X)\|_{\mathbb{H}}^{2}=2 \mathcal{E}(F, F) .
\end{aligned}
$$

Acknowledgment. The author would like to thank the referee for helpful comments and corrections.

\section{REFERENCES}

[1] J.M. Bismut, The calculus of boundary processes, Ann. Sci. École Norm. Sup., 17, 507-622, 1984.

[2] M. Capitaine, E.P. Hsu, and M. Ledoux, Martingale representation and a simple proof of logarithmic Sobolev inequalities on path spaces, Electron Commun. Prob., 2, 71-81, 1997.

[3] B.K. Driver, A Cameron-Martin type quasi-invariance theorem for Brownian motion on a compact Riemannian manifold, J. Funct. Anal., 110, 272-376, 1992. 
[4] K.D. Elworthy and X.M. Li, An $L^{2}$ theory for differential forms on path spaces I, J. Funct. Anal., 254, 196-245, 2008.

[5] S. Fang, Inegalité du type de Poincaré sur l'espace des chemins riemanninens, C.R. Acad. Sci. Paris, 318, 257-260, 1994.

[6] L. Gross, Logarithmic Sobolev inequalities, Amer. J. Math., 97, 1061-1083, 1976.

[7] S. Fang and P. Malliavin, Stochastic analysis on the path space of a Riemannian manifold, J. Funct. Anal., 118, 249-274, 1993.

[8] E.P. Hsu, Logarithmic-Sobolev inequalities on path spaces over Riemannian manifolds, Commun. Math. Phys., 189, 9-16, 1997.

[9] E.P. Hsu, Multiplicative functional for the heat equation on manifolds with boundary, Michigan Math. J., 50, 351-367, 2002.

[10] P. Malliavin, Stochastic Analysis, Springer, New York, 1997.

[11] D. Stroock, An Introduction to the Analysis of Paths on a Riemannian Manifold, Amer. Math. Soc., 2000.

[12] L. Zambotti, Integration by parts on the law of the reflecting Brownian motion, J. Funct. Anal. $223,147-178,2005$. 